\title{
Qualidade de vida de trabalhadores de instituição de longa permanência para
}

\section{idosos: revisão de escopo}

\author{
Quality of life of workers in a long stay institution for the elderly: scoping review \\ Calidad de vida de los trabajadores en una institución de larga estancia para mayores: revisión del
} alcance

Fernanda Seidel Anastácio

ORCID: https://orcid.org/0000-0002-9018-8312 Universidade Federal do Rio Grande do Sul, Brasil,

E-mail: fernandaseidelp@gmail.com

Ana Karina Silva da Rocha Tanaka

ORCID: https://orcid.org/0000-0003-2488-3656

Universidade Federal do Rio Grande do Sul, Brasi E-mail: anakarinatanaka@gmail.com

Cecília Helena Glanzner

ORCID: https://orcid.org/0000-0002-2553-8582 Universidade Federal do Rio Grande do Sul, Brasil E-mail: glanznercecilia@gmail.com

Carlise Rigon Dalla Nora

ORCID: https://orcid.org/0000-0001-5501-2146 Universidade Federal do Rio Grande do Sul, Brasil E-mail: carliserdn@gmail.com

Lisiane Paula Sordi Matzenbacher ORCID: https://orcid.org/0000-0002-1407-7717 Hospital de Clínicas de Porto Alegre, Brasil E-mail: 1sordi@hcpa.edu.br

Rosaura Soares Paczek

ORCID: https://orcid.org/0000-0002-4397-1814 Hospital de Clínicas de Porto Alegre, Brasil E-mail: rspaczek@gmail.com

\section{Resumo}

Objetivo: analisar os fatores que influenciam a qualidade de vida dos trabalhadores de instituições de longa permanência para idosos. Métodos: revisão de escopo com seleção de artigos sobre o tema nas bases SciELO, Pubmed e CINAHL, no período de agosto a outubro de 2020. Foram incluídos estudos com trabalhadores de instituições de longa permanência para idosos. Para a análise dos dados, utilizou-se um instrumento no Excel para categorizar e melhor visualizar o relatório dos elementos essenciais. Resultados: conforme a busca nas bases de dados foram encontrados 143 artigos, sendo incluídos 8 artigos. Os dados foram classificados em três categorias: caracterização dos trabalhadores, fatores que influenciam na qualidade de vida dos trabalhadores de instituição de longa permanência para idosos e estratégias de enfrentamento. Conclusão: conclui-se que esta pesquisa é relevante e contribui tanto para o trabalhador quanto para os idosos das instituições de longa permanência, no momento em que trará o olhar das demandas e dificuldades encontradas no seu exercício profissional.

Palavras-chave: Instituição de longa permanência para idosos; Saúde do trabalhador; Qualidade de vida.

\begin{abstract}
Objective: to analyze the factors that influence the quality of life of workers in long-stay institutions for the elderly. Methods: scope review with selection of articles on the subject in the SciELO, Pubmed and CINAHL databases, from August to October 2020. Studies with workers from long-stay institutions for the elderly were included. For data analysis, an Excel instrument was used to categorize and better visualize the essential elements report. Results: according to the search in the databases, 143 articles were found, including 8 articles. Data were classified into three categories: characterization of workers, factors that influence the quality of life of workers in a long-term care institution for the elderly, and coping strategies. Conclusion: it is concluded that this research is relevant and contributes to both the worker and the elderly in long-stay institutions, at a time when it will bring a look at the demands and difficulties encountered in their professional practice.
\end{abstract}

Keywords: Homes for the aged; Occupational health; Quality of life. 


\begin{abstract}
Resumen
Objetivo: analizar los factores que influyen en la calidad de vida de los trabajadores en instituciones de larga estancia para ancianos. Métodos: revisión de alcance con selección de artículos sobre el tema en las bases de datos SciELO, Pubmed y CINAHL, de agosto a octubre de 2020. Se incluyeron estudios con trabajadores de instituciones de larga estancia para ancianos. Para el análisis de datos se utilizó un instrumento Excel para categorizar y visualizar mejor el reporte de elementos esenciales. Resultados: de acuerdo con la búsqueda en las bases de datos, se encontraron 143 artículos, incluidos 8 artículos. Los datos se clasificaron en tres categorías: caracterización de los trabajadores, factores que influyen en la calidad de vida de los trabajadores en una institución de atención a largo plazo para ancianos y estrategias de afrontamiento. Conclusión: se concluye que esta investigación es relevante y aporta tanto al trabajador como al anciano en instituciones de larga estancia, en un momento en el que traerá una mirada a las demandas y dificultades encontradas en su práctica profesional.
\end{abstract}

Palabras clave: Hogares para ancianos; Salud laboral; Calidad de vida.

\title{
1. Introdução
}

A maior conquista do século XX foi chegar à velhice, anteriormente era privilégio de poucos, sendo um desafio do século atual. Esta expansão do tempo de vida é considerada um dos grandes feitos da humanidade, acompanhada da melhoria dos parâmetros de saúde das populações. Viver mais é ilustre desde que seja possível associar qualidade aos anos adicionais de vida (Veras \& Oliveira, 2018).

O envelhecimento é um movimento natural e desejável a todos os seres vivos, iniciando no nascimento, mudanças complexas acontecem gradualmente, tanto biológicas quanto fisiológicas e psíquicas, atualmente na sociedade ocidental, o ser humano parece desprezá-lo, parece rejeitar a existência de pessoas idosas e acabam negligenciando as demandas especiais daqueles que atingiram mais de sessenta anos (Brasil, 2016).

As Instituições de Longa Permanência para Idosos (ILPI), anteriormente ligadas à filantropias de cunho religioso, prestam serviços voluntários a pessoas carentes, desamparadas, em situação de pobreza, de diversas idades, necessitando de atenção à saúde e abrigo. Acarretando uma imagem negativa, reforçada por cuidados de baixa qualidade, remetendo ao sentimento de solidão e abandono (Alves et al., 2017).

As ILPIs são residenciais para moradia comunitária de indivíduos com 60 anos ou mais, com ou sem suporte familiar. E referente a organização do serviço, destaca-se o grau de dependência, capacidades de locomoção e autocuidado pelos internos (ANVISA, 2005).

No Brasil, as instituições de cuidado ao idoso eram conhecidas como asilos, palavra frequentemente associada a cenários negativos e preconceituosos e de renúncia dos idosos pelos familiares. Entretanto, a perceptível redução da disponibilidade do cuidado familiar tradicional indica uma necessidade maior de cuidados formais. Neste caso, os modelos alternativos de cuidados a idosos demonstram-se grandes cúmplices (Barbosa et al., 2017).

O modo que o residencial é vivido depende diretamente do paciente, enfrentando os preconceitos, das normas culturais e das relações familiares. Compete à ILPI oferecer uma assistência geriátrica e gerontológica dirigida para as necessidades dos seus clientes, dotadas de equipes multiprofissionais qualificadas para exercer o trabalho (Oliveira \& Tavares, 2014).

A saúde do trabalhador corresponde a um domínio da saúde pública, reunido a atividade laboral, destacando a proteção e promoção da saúde do trabalhador. Devido a mudanças no mercado de trabalho e elevação da expectativa de vida, há maior necessidade de promoção da saúde dos trabalhadores, especialmente na prevenção e controle de sintomas osteomusculares decorrentes de agravos na saúde física e qualidade de vida (Mendes et al., 2017; Vasconcelos et al., 2017; Amorim et al., 2018).

Segundo a Organização Mundial da Saúde (2012), a qualidade de vida (QV) é compreendida como a percepção que o indivíduo tem de sua posição na vida, no enredo da cultura e sistema de valores nos quais vive, e em relação aos seus objetivos, expectativas, padrões e inquietações (Pimenta et al.,2019).

Presume-se que esta pesquisa é relevante e contribui tanto para o trabalhador quanto para os idosos das ILPI, trazendo 
o olhar das demandas e dificuldades encontradas no seu exercício profissional. Objetivou-se analisar os fatores que influenciam a qualidade de vida dos trabalhadores de instituições de longa permanência para idosos.

\section{Metodologia}

Trata-se de uma revisão de escopo, sendo utilizados os seguintes passos: Identificação da questão de investigação; Identificação dos estudos relevantes; Mapeamento dos dados dos estudos incluídos na revisão; Resumir e reportar os resultados da revisão (Arksey \& O'malley, 2005).

A coleta de dados aconteceu entre agosto e outubro de 2020 por meio da revisão, síntese e leitura dos artigos selecionados. Utilizou-se a estratégia PCC para formulação da pergunta, sendo "P" para população/participantes (trabalhadores), "C" para o conceito que se pretende investigar (qualidade de vida), "C" para contexto (instituições de longa permanência para idosos). Assim a questão norteadora foi: Quais fatores influenciam na qualidade de vida dos trabalhadores de Instituições de Longa Permanência para Idosos?

Os critérios de inclusão foram: artigos originais, relatos de experiência e estudos de caso que caracterizassem a saúde e qualidade de vida dos trabalhadores de ILPIs, nos idiomas Português, Inglês e Espanhol, textos completos e de acesso gratuito. Os critérios de exclusão foram: estudos que não respondessem à questão norteadora, como trabalhadores da área hospitalar ou da atenção básica, ou até mesmo estudos que abordassem a qualidade de vida dos idosos institucionalizados e não dos trabalhadores em si.

A busca foi desenvolvida nas bases de dados Scientific Electronic Library Online (SCIELO), National Library of Medicine (PubMed), e CINAHL, utilizando a estratégia de pesquisa booleana com os seguintes descritores: Saúde do trabalhador (occupational health); Qualidade de vida (quality of life); Instituições de Longa Permanência para Idosos; ILPI's; Lar de idosos; Asilo; Casa de repouso (Homes for the Aged), utilizados isoladamente e associados conforme características das bases de dados, nos idiomas Português, Inglês e Espanhol, textos completos e de acesso gratuito. As associações realizadas foram: "instituição de longa permanência para idosos" AND "saúde do trabalhador" AND "qualidade de vida", tais associações foram replicadas em inglês e espanhol.

As referências dos estudos incluídos, também foram verificadas com o intuito de localizar outras pesquisas que poderiam ser relevantes para a temática desta revisão.

Foi criado um instrumento no Excel para resumir e reportar os resultados, com categorização e melhor visualizar o relatório dos elementos essenciais, propiciando identificação dos estudos, como: autor/ano, objetivos, população, tipo de estudo, métodos e fatores relacionados à qualidade de vida. Organizou-se um quadro sinóptico geral a fim de orientar os resultados da pesquisa, atribuindo uma numeração para as publicações encontradas.

Os resultados foram apresentados por meio de uma síntese numérica e temática. Na síntese numérica, serão descritas as características dos estudos incluídos. Já a síntese temática será organizada de acordo com a natureza dos fatores que influenciam a qualidade de vida dos trabalhadores de ILPI, gerando uma apresentação ampla sobre o tema.

Os aspectos éticos foram respeitados conforme a Lei $\mathrm{n}^{\circ}$ 12.853, de 14 de agosto de 2013, e todos os autores foram propriamente citados conforme indicado (Brasil, 2013).

\section{Resultados e Discussão}

Na presente scoping review foram encontrados 143 artigos a partir das bases de dados selecionadas, destes 137 foram excluídos após leitura do título e resumo, sendo destes 10 excluídos por serem artigos repetidos e 02 foram excluídos após leitura completa do artigo. Acrescentou-se mais 02 artigos através da pesquisa manual, totalizando 08 artigos incluídos na amostra. 
Research, Society and Development, v. 10, n. 14, e429101422022, 2021

(CC BY 4.0) | ISSN 2525-3409 | DOI: http://dx.doi.org/10.33448/rsd-v10i14.22022

Na Figura 1, pode-se visualizar melhor o processo de análise, inclusão e exclusão dos artigos desta amostra, conforme orientado pela JBI (Arksey \& O'malley, 2005).

Figura 1. Fluxograma referente ao processo de busca dos artigos na literatura, adaptado do PRISMA.

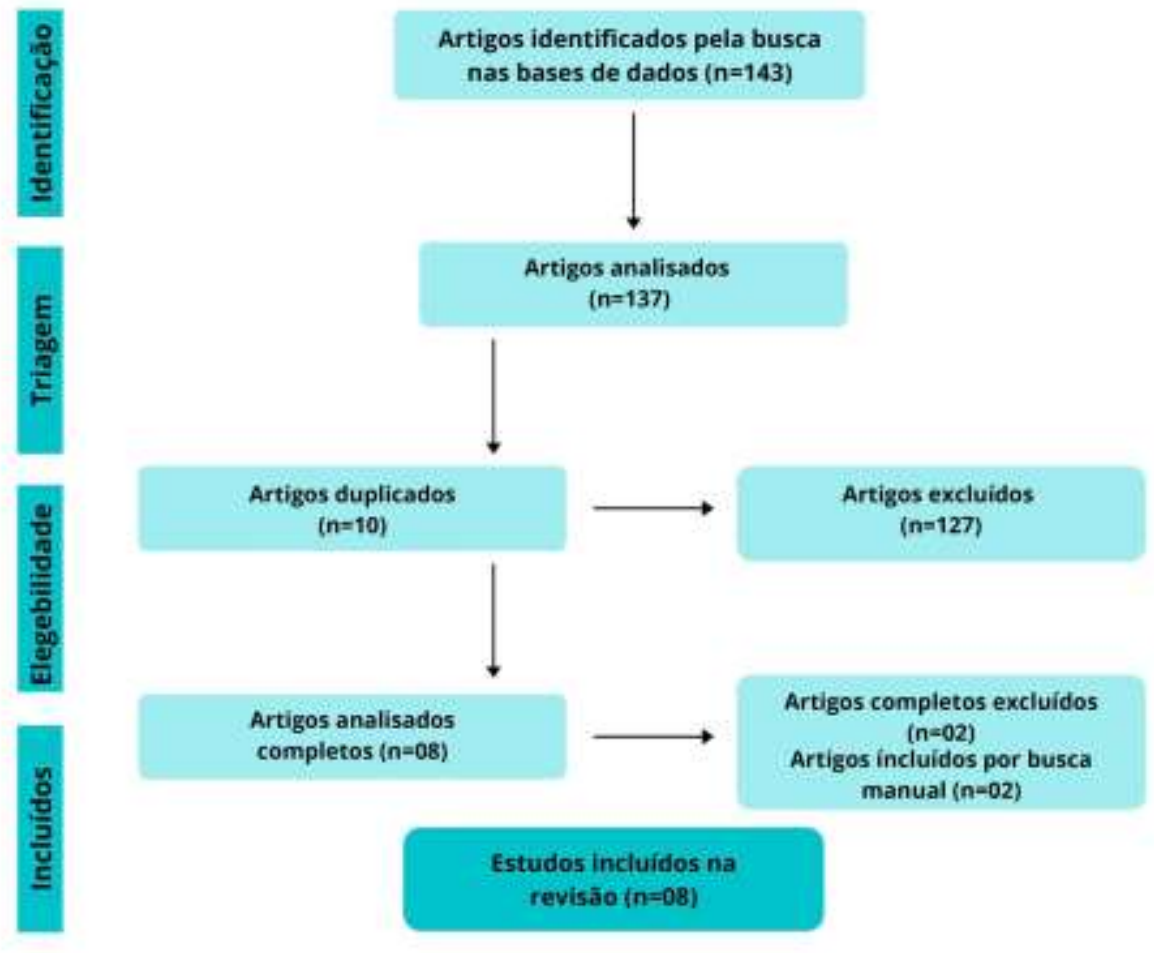

Fonte: Autores.

No Quadro 1, apresenta-se os artigos incluídos na amostra e algumas características como, autor e ano de publicação, objetivos, população, tipo de estudo, métodos e fatores relacionados à qualidade de vida. 
Quadro 1. Quadro sinóptico que caracteriza os artigos incluídos nesta amostra.

\begin{tabular}{|c|c|c|c|c|c|c|}
\hline $\mathbf{N}^{\mathbf{o}}$ & Autor/Ano & Objetivos & População & Tipo de estudo & Métodos & Fatores relacionados à qualidade de vida \\
\hline 01 & $\begin{array}{l}\text { Mariano PP, } \\
\text { Carreira L. } \\
\text { (2016) }\end{array}$ & $\begin{array}{c}\text { Descrever as } \\
\text { estratégias } \\
\text { defensivas utilizadas } \\
\text { pelos trabalhadores } \\
\text { de enfermagem das } \\
\text { ILPI perante o } \\
\text { sofrimento laboral. }\end{array}$ & \begin{tabular}{|c} 
\\
\\
5 \\
Enfermeiras, \\
12 técnicos de \\
enfermagem e \\
10 auxiliares \\
de \\
enfermagem, \\
sendo 23 do \\
sexo \\
feminino.
\end{tabular} & $\begin{array}{c}\text { Pesquisa } \\
\text { descritiva e } \\
\text { exploratória, } \\
\text { com abordagem } \\
\text { qualitativa, } \\
\text { originado de } \\
\text { dissertação. }\end{array}$ & $\begin{array}{l}\text { Ao final das entrevistas, fizeram } \\
\text { parte da pesquisa } 27 \text { trabalhadores } \\
\text { de enfermagem das seis ILPI. }\end{array}$ & $\begin{array}{l}\text { Uma estratégia de adaptação e exploração } \\
\text { identificada nos discursos dos profissionais foi } \\
\text { a não criação de vínculo com os idosos, } \\
\text { desenvolvendo assim uma barreira emocional } \\
\text { diante do sofrimento gerado com a morte dos } \\
\text { pacientes e evitando prejuízos no desempenho } \\
\text { do trabalho. Os profissionais se referem à } \\
\text { convivência com situações geradoras de } \\
\text { sofrimento, como a incapacidade de curar ou } \\
\text { aliviar as dores e desconfortos dos idosos, que } \\
\text { pioram gradativamente, evoluindo a óbito. } \\
\text { Estes trabalhadores de enfermagem, mesmo } \\
\text { experenciando sentimentos negativos como } \\
\text { tristeza e desânimo frente estes } \\
\text { acontecimentos, não deixam se abalar, devido } \\
\text { às responsabilidades que possuem frente os } \\
\text { demais idosos. Neste sentido, os trabalhadores } \\
\text { convivem com o sofrimento, transpassando-o e } \\
\text { atingindo uma condição de normalidade na } \\
\text { realização da sua atividade laboral. }\end{array}$ \\
\hline 02 & $\begin{array}{c}\text { Colomé ICS, } \\
\text { Marqui ABT, } \\
\text { Jahn AC, } \\
\text { Resta DG, } \\
\text { Carli R, } \\
\text { Winck MT } \\
\text { et al. (2011) }\end{array}$ & $\begin{array}{c}\text { Conhecer as } \\
\text { características e o } \\
\text { trabalho de } \\
\text { cuidadores de idosos } \\
\text { institucionalizados, } \\
\text { identificando as } \\
\text { dificuldades } \\
\text { enfrentadas por eles } \\
\text { no seu cotidiano. }\end{array}$ & 11 cuidadores & $\begin{array}{l}\text { Estudo } \\
\text { exploratório } \\
\text { descritivo }\end{array}$ & $\begin{array}{c}\text { Aplicação de questionário } \\
\text { semiestruturado composto por } \\
\text { questões fechadas e abertas. As } \\
\text { primeiras eram relativas à } \\
\text { caracterização dos cuidadores } \\
\text { (sexo, idade, estado civil, } \\
\text { escolaridade) e as questões abertas } \\
\text { relacionadas ao trabalho } \\
\text { desenvolvido na instituição e suas } \\
\text { principais dificuldades } \\
\text { (treinamento para cuidar dos } \\
\text { idosos, jornada de trabalho, } \\
\text { atividades desenvolvidas na } \\
\text { instituição, dificuldades no } \\
\text { trabalho, sugestões de melhoria, } \\
\text { interesse em capacitações, entre } \\
\text { outras). }\end{array}$ & $\begin{array}{l}\text { A partir das dificuldades referidas pelos } \\
\text { cuidadores no cuidado aos idosos, foi possível } \\
\text { a construção de duas subcategorias temáticas: } \\
\text { "sobrecarga de trabalho e exigência física" e } \\
\text { "necessidade de conhecimento para cuidar dos } \\
\text { idosos" Segundo os cuidadores a sobrecarga de } \\
\text { trabalho comprometeu a prática de um cuidado } \\
\text { adequado aos idosos, especialmente, àqueles } \\
\text { que apresentaram alguma patologia, por } \\
\text { necessitarem de uma atenção maior e } \\
\text { assistência diferenciada. O presente estudo } \\
\text { mostrou que os cuidadores, exceto a auxiliar } \\
\text { de enfermagem, exercem as mais diversas } \\
\text { atividades, sem uma definição clara da função } \\
\text { de cada um na equipe. Essa realidade pode } \\
\text { ocasionar uma sobrecarga de trabalho, já que } \\
\text { não existe uma distribuição equitativa das } \\
\text { funções de cada trabalhador. }\end{array}$ \\
\hline
\end{tabular}




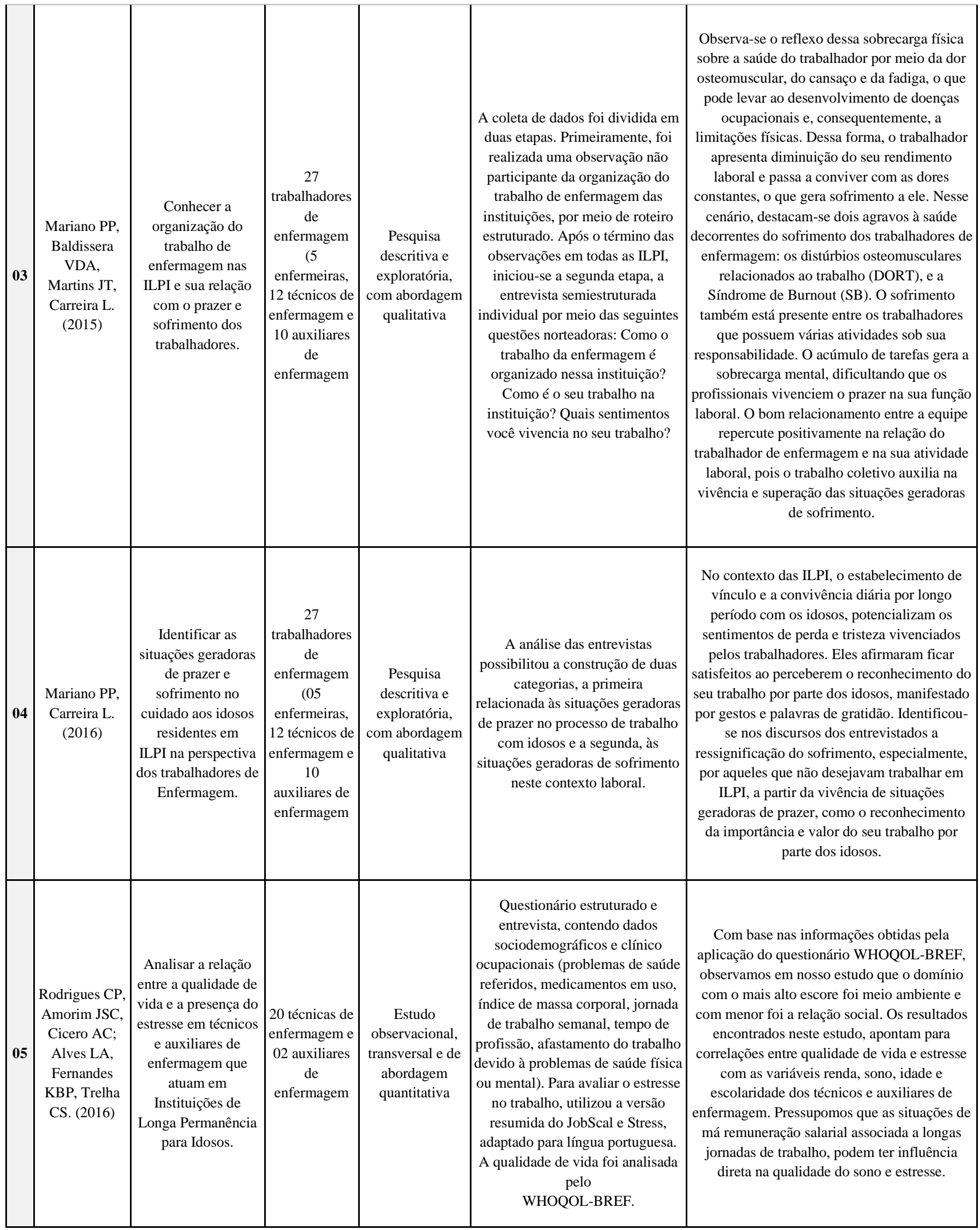




\begin{tabular}{|c|c|c|c|c|c|c|}
\hline 06 & $\begin{array}{l}\text { Santos SMC, } \\
\text { Oliveira LS, } \\
\text { Santos MCS, } \\
\text { Carneiro SR, } \\
\text { Santos LLS, } \\
\text { Teodori RM. } \\
\text { (2017) }\end{array}$ & $\begin{array}{l}\text { Avaliar a sobrecarga } \\
\text { de trabalho e a } \\
\text { qualidade de } \\
\text { vida de cuidadores } \\
\text { formais de idosos } \\
\text { institucionalizados, } \\
\text { com vistas a } \\
\text { conhecer suas } \\
\text { dificuldades e, } \\
\text { futuramente, propor } \\
\text { estratégias de } \\
\text { prevenção e proteção } \\
\text { da saúde destes } \\
\text { profissionais. }\end{array}$ & 12 cuidadores & $\begin{array}{c}\text { Tipo inquérito } \\
\text { institucional e } \\
\text { descritivo, de } \\
\text { caráter } \\
\text { quantitativo e } \\
\text { de corte } \\
\text { transversal }\end{array}$ & $\begin{array}{l}\text { 1) Questionário de caracterização } \\
\text { do cuidador, o qual apresenta } 14 \\
\text { perguntas que envolvem aspectos } \\
\text { sociais e econômicos e que } \\
\text { caracterizam a função do } \\
\text { cuidador; 2) Escala de Sobrecarga } \\
\text { do Cuidador de Zarit, a qual conta } \\
\text { com } 22 \text { questões e encontra-se } \\
\text { validada e traduzida no Brasil, } \\
\text { cujo objetivo é identificar os } \\
\text { fatores que levam à exaustão do } \\
\text { cuidador. 3) Questionário de } \\
\text { Qualidade de Vida WHOQOL- } \\
\text { bref. }\end{array}$ & $\begin{array}{c}\text { Neste estudo, a maioria dos cuidadores de } \\
\text { idosos institucionalizados era do gênero } \\
\text { masculino, diferente do observado por diversos } \\
\text { outros estudos nos quais predomina o gênero } \\
\text { feminino. Os Escores da Escala de Sobrecarga } \\
\text { do Cuidador de Zarit do presente estudo } \\
\text { estavam divididos entre ausência de sobrecarga } \\
\text { e sobrecarga moderada a leve. Em relação ao } \\
\text { resultado do questionário de qualidade de vida } \\
\text { WHOQOL-Bref, no presente estudo, os } \\
\text { maiores escores estavam relacionados aos } \\
\text { domínios relações sociais, psicológico e físico. }\end{array}$ \\
\hline 07 & $\begin{array}{l}\text { Andrade } \\
\text { SMB, } \\
\text { Reis LA. } \\
(2018)\end{array}$ & $\begin{array}{l}\text { Averiguar as } \\
\text { repercussões do } \\
\text { toque terapêutico na } \\
\text { qualidade de vida } \\
\text { dos cuidadores } \\
\text { formais de idosos, e } \\
\text { por objetivos } \\
\text { específicos } \\
\text { caracterizar as } \\
\text { condições } \\
\text { sociodemográficas } \\
\text { de cuidadores } \\
\text { formais de idosos e } \\
\text { traçar o perfil de } \\
\text { saúde deles. }\end{array}$ & $\begin{array}{l}4 \text { Cuidadoras } \\
\text { do sexo } \\
\text { feminino }\end{array}$ & $\begin{array}{c}\text { Pesquisa } \\
\text { exploratória } \\
\text { descritiva do tipo } \\
\text { experimental e } \\
\text { abordagem } \\
\text { quantitativa }\end{array}$ & $\begin{array}{l}\text { O instrumento da pesquisa foi } \\
\text { constituído de dados } \\
\text { sociodemográficos } \\
\text { e uso do Whoqol-bref. Os dados } \\
\text { foram analisados de forma } \\
\text { descritiva por meio do Programa } \\
\text { Estatístico SPSS Versão 21.0. }\end{array}$ & $\begin{array}{l}\text { Ao analisar os resultados obtidos no presente } \\
\text { estudo, pôde-se perceber que houve uma } \\
\text { melhora na qualidade de vida dos cuidadores } \\
\text { de idosos após o toque terapêutico. Observa-se } \\
\text { na tabela I que, na avaliação da qualidade de } \\
\text { vida antes do toque terapêutico, os domínios } \\
\text { de menores médios foram os domínios } \\
\text { psicológico e meio ambiente. Após a } \\
\text { realização do toque terapêutico verificou-se na } \\
\text { avaliação da qualidade de vida que os } \\
\text { domínios de menores médias foram os } \\
\text { domínios psicológico ( } 66,40 \pm 8,20 \text { pontos) e } \\
\text { meio ambiente ( } 72,92 \pm 12,96 \text { pontos). }\end{array}$ \\
\hline 08 & $\begin{array}{l}\text { Lopes LA, } \\
\text { Mitre } \\
\text { NCD, Coelho } \\
\text { MACM. } \\
\text { (2012) }\end{array}$ & $\begin{array}{c}\text { Descrever o perfil } \\
\text { sociodemográfico } \\
\text { dos cuidadores das } \\
\text { ILPIs do Município } \\
\text { de Itaúna (MG); } \\
\text { avaliar a relação } \\
\text { entre sintomas } \\
\text { depressivos e } \\
\text { qualidade de vida e } \\
\text { analisar a relação } \\
\text { entre sintomas } \\
\text { depressivos e nível } \\
\text { de ansiedade nessa } \\
\text { população. }\end{array}$ & $\begin{array}{c}25 \\
\text { Cuidadores }\end{array}$ & $\begin{array}{c}\text { Estudo } \\
\text { observacional } \\
\text { exploratório } \\
\text { transversal }\end{array}$ & $\begin{array}{l}\text { Os instrumentos utilizados foram: } \\
\text { entrevista estruturada } \\
\text { sociodemográfica, } \\
\text { avaliação da qualidade de vida } \\
\text { através do instrumento Medical } \\
\text { Outcomes Short Form Health } \\
\text { Survey (SF-36), ansiedade através } \\
\text { do Inventário de Ansiedade Traço- } \\
\text { Estado (IDATE) e sintomas } \\
\text { depressivos por meio do } \\
\text { Inventário de Depressão de Beck } \\
\text { (IDB). }\end{array}$ & $\begin{array}{l}\text { Por meio dos dados apresentados percebe-se } \\
\text { que é alta a prevalência de sintomas } \\
\text { depressivos entre os cuidadores (48\%), e que } \\
\text { quanto mais sintomas depressivos eles } \\
\text { apresentam, maior é o prejuízo nos } \\
\text { subdomínios da qualidade de vida, capacidade } \\
\text { funcional, aspecto físico, dor, estado geral de } \\
\text { saúde, vitalidade e saúde mental, verificado } \\
\text { pelo SF-36. Neste trabaho também, foi } \\
\text { demonstrado que quanto maiores os sintomas } \\
\text { depressivos maiores os índices de ansiedade } \\
\text { (Traço e Estado). }\end{array}$ \\
\hline
\end{tabular}

Fonte: Autores (2021)

Os artigos incluídos em sua totalidade são de origem brasileira, distribuindo-se nos anos 2011 e 2020, adaptado conforme proposto pela revisão de escopo. A justificativa de acrescentar estudos fora dos últimos cinco anos ocorre devido à escassez de estudos abordando a temática proposta. Dos estudos encontrados $50 \%$ são referentes somente a cuidadores e os outros $50 \%$ composto por enfermeiros, técnicos e auxiliares de enfermagem. A maioria dos estudos $(\mathrm{n}=5)$ utilizaram metodologia quantitativa e $(n=2)$ qualitativa.

A seguir, apresenta-se as três categorias evidenciadas a partir da análise dos estudos selecionados: caracterização dos trabalhadores, fatores que influenciam na qualidade de vida dos trabalhadores de instituição de longa permanência para idosos e estratégias de enfrentamento. 


\subsection{Caracterização dos trabalhadores, tarefas e ambiente de ILPI}

Analisando a amostra, nota-se que há prevalência de estudos que abordam a equipe de enfermagem, especialmente a classe de cuidadores. Deixando a desejar saber sobre as outras categorias profissionais nas ILPI.

As ILPI são ambientes laborais que produzem cargas negativas aos trabalhadores de enfermagem, bem como outros locais de trabalho desta classe profissional (Mariano \& Carreira, 2016). Entre as tarefas realizadas no cuidado aos idosos estão a preparação de alimentos e auxílio na alimentação, limpeza do local e higienização dos idosos (troca de fraldas, banho), lavagem, costura e organização das roupas, aferição de pressão arterial, realização de curativos, controle e administração de medicamentos, acompanhamento em consultas realização de exames clínicos, manutenção do prédio e do mobiliário, assim como cuidado com o jardim e a horta (Colomé et al., 2011).

O trabalho de enfermagem em ILPI é entendido como uma ocupação difícil sob diversos aspectos, mas especialmente quando percebido a partir das relações humanas estabelecidas na continuidade do cuidado, nas quais vários idosos adotam comportamentos resistentes às prescrições de seus cuidados (Colomé et al., 2011).

À vista disso, é indispensável a análise do ambiente e da organização do trabalho das ILPI, dado que os trabalhadores de enfermagem também estão suscetíveis a doenças ocupacionais (Mariano \& Baldissera, 2015).

\subsection{Fatores que influenciam na qualidade de vida dos trabalhadores de ILPI}

Um fator que influenciou na qualidade de vida dos trabalhadores identificadas nos estudos foi o prazer. A formação de vínculos afetivos entre idosos e trabalhadores, ao passo que estes vão se intensificando, ocorre uma troca do relacionamento puramente profissional para uma relação familiar. Assim, o trabalho torna-se fonte de alegria e satisfação, pois gera o sentimento de prazer. Trabalhadores alegam ficar satisfeitos ao identificarem o reconhecimento do seu trabalho por parte dos idosos, revelado através de gestos e palavras de gratidão (Mariano \& Baldissera, 2015).

A maneira como o trabalho de enfermagem é elaborado nas ILPI possibilita que os trabalhadores exerçam algo além das suas obrigações, permitindo que o seu trabalho seja gerador de prazer (Mariano \& Carreira, 2016).

E a partir do depoimento dos trabalhadores, ficam claros os sentimentos de orgulho e utilidade quando contam a sua contribuição para o conforto, alívio da dor e avanço do estado de saúde dos idosos (Mariano \& Baldissera, 2015).

Outro fator que influenciou a qualidade de vida dos trabalhadores foi a sobrecarga de trabalho. Os cuidadores responsáveis por 36 idosos referiram que o excesso de atribuições tem como consequência a carência de tempo para realizar as atividades intrínsecas à sua ocupação, o que gera impacto direto na assistência prestada, sendo prejudicial a eles a aos idosos (Colomé et al., 2011).

Outra justificativa possível para essa sobrecarga é a questão da dependência do idoso, constantemente incentivado pelo cuidador. Pois, existe a predisposição do não estímulo de suas capacidades para progredir nas atividades básicas da vida diária, o que acaba suscitando diminuição da autonomia do idoso (Colomé et al., 2011).

Deduziram que as longas jornadas de trabalho e as situações de má remuneração salarial podem atuar de forma direta na qualidade do sono e estresse dos trabalhadores. Corrobora com este estudo que a sobrecarga de trabalho também pode estar associada devido à escassez de recursos humanos para operar na ILPI (Rodrigues et al., 2016; Colomé et al., 2011).

Conforme apresentaram os Escores da Escala de Sobrecarga do Cuidador de Zarit, que estavam classificados entre ausência de sobrecarga e sobrecarga moderada a leve. O maior número dos cuidadores que colaborou com este estudo demonstrou sobrecarga moderada a leve (Santos et al., 2016).

Com os dados obtidos através do questionário WHOQOL-BREF, um dos estudos apontou que o domínio com maior escore foi o meio ambiente e o menor foi a relação social. Em outro estudo, com o mesmo questionário, apresentou nos resultados que maiores escores eram relacionados aos domínios relações sociais, psicológico e físico (Rodrigues et al., 2016; Santos et al., 
2016).

A morte dos idosos, foi outro fator que influenciou na qualidade de vida dos trabalhadores. Os profissionais respondem de maneiras diferentes diante do óbito. Na mesma proporção que alguns consideram a morte como algo previsto e natural, outros vivenciam o sentimento de tristeza que chega a interferir na sua saúde, no tempo de superação e regresso à rotina de trabalho (Veras \& Oliveira, 2018).

Ao racionalizar a morte dos idosos como algo natural, os trabalhadores de enfermagem ainda demonstram sentimentos de tristeza a partir do ocorrido, comprovando que mesmo utilizando esta estratégia de enfrentamento, os trabalhadores seguem vivenciando situações de sofrimento (Mariano \& Carreira, 2016).

O desgaste físico também é um fator que influencia a qualidade de vida dos trabalhadores. O ritmo apressado da rotina de trabalho, onde as tarefas devem ser executadas dentro de um limite de tempo, com a intenção de não comprometer as ações posteriores, provoca frustração e insatisfação nos trabalhadores como resultado do seu trabalho. A pressão pela realização cronometrada das atividades, analisada em todas as instituições, impulsiona a relação de sofrimento desses profissionais com o trabalho (Rodrigues et al., 2016).

Visto que muitas das atividades desenvolvidas pelos cuidadores exigiam força física, os pesquisados relataram estar apresentando problemas de saúde como dores na coluna, provavelmente decorrentes de postura inadequada e uso da força muscular (Colomé et al., 2011).

Neste contexto, destacam-se dois agravos relacionados à saúde ocupacional decorrentes do sofrimento dos trabalhadores de enfermagem, distúrbios osteomusculares relacionados ao trabalho (DORT), e a Síndrome de Burnout (SB) (Rodrigues et al., 2016).

Os cuidadores informaram que as tarefas que mais geram desgaste físico eram auxiliar nas mudanças posturais, dar banho e acompanhar os idosos que estavam internados nos hospitais. É interessante ressaltar que na hospitalização dos idosos, os cuidadores eram transferidos para o hospital, reduzindo o número de trabalhadores presentes na instituição, gerando sobrecarga (Rodrigues et al., 2016). É considerado uma das características da sobrecarga física no trabalho, quando trabalhadores realizam esforço físico expressivo durante a higienização e mobilização dos idosos (Veras \& Oliveira, 2018).

A sobrecarga mental nos trabalhadores foi mais um fator que influenciou a qualidade de vida. $\mathrm{O}$ acúmulo de atividades dificulta que os trabalhadores vivenciam o prazer no seu exercício laboral, gerando sobrecarga mental. Diversos encargos sob o comprometimento de um trabalhador são comumente observados no cenário da enfermagem, principalmente sobre o enfermeiro. Desse modo, estes profissionais passam a manifestar sentimentos de desprazer, ansiedade e insatisfação (Rodrigues et al., 2016).

$\mathrm{Na}$ equipe de enfermagem, o enfermeiro ocupa o cargo que apresenta maior conhecimento científico para lidar com situações de urgência e emergência das ILPI. Porém, em duas das instituições pesquisadas, são os técnicos e auxiliares de enfermagem que assumem a condução, por não possuírem enfermeiro na escala de funcionários. Essa realidade produz divergências entre a equipe e desgaste psicológico à vista das medidas que devem ser tomadas (Rodrigues et al., 2016).

Os cuidadores, participantes do estudo em Itaúna, apresentaram alta da prevalência de sintomas depressivos (48\%). E que quanto maior expressão de sintomas depressivos, mais elevado prejuízo nos subdomínios da qualidade de vida, capacidade funcional, aspecto físico, dor, estado geral de saúde, vitalidade e saúde mental, averiguado pelo SF-36. Nesta pesquisa também, foi comprovado que quanto mais elevado os sintomas depressivos maiores os índices de ansiedade (Traço e Estado) (Lopes et al., 2012).

O sofrimento laboral é um fator que influencia a qualidade de vida dos trabalhadores. Algumas situações que originam sofrimento nos trabalhadores de ILPI em relação ao seu trabalho com idosos, destacam-se o testemunhar o distanciamento e desinteresse dos familiares pelo idoso. Bem como o estabelecer vínculo e o conviver diariamente por longos períodos com os idosos, fortalecem sentimentos de perda e tristeza experienciados pelos trabalhadores (Mariano \& Baldissera, 2015). 


\subsection{Estratégias de enfrentamento}

Uma estratégia de enfrentamento evidenciada nos estudos é o toque terapêutico. É uma prática holística por diversas abordagens antepassadas onde a partir das mãos se realiza o papel essencial da prática. Auxilia no alívio de dores, proporciona conforto, melhora ansiedade e humor e diminui o estresse (Andrade \& Reis, 2018).

O método utiliza seis sessões de massagem altamente relaxantes com auxílio de creme de massagem corporal, além de aplicação de música tranquilizante durante o procedimento. Comprovando que após a utilização do toque terapêutico houve melhora na qualidade de vida dos cuidadores de idosos (Andrade \& Reis, 2018).

Outra estratégia foi a racionalização do sofrimento. Adota-se a racionalização do sofrimento frente à morte do idoso no momento em que se considera um aspecto natural do ciclo de vida, nota-se a estratégia tal como barreira de proteção pelos trabalhadores de enfermagem, ou seja, existe a ideia compensatória a respeito da morte do idoso (Mariano \& Carreira, 2016).

E outro meio de racionalizar o sofrimento é constatada na presença do óbito de um idoso que estava em situação crítica e que lhe provocava sofrimento. Neste caso, a morte é repensada pelos trabalhadores como forma de término desta situação dolorosa, despertando um sentimento de alívio, não apenas ao idoso, mas também para quem estava envolvido nessa conjunção (Mariano \& Carreira, 2016).

Em relação às estratégias de adaptação e exploração utilizadas pelos profissionais, identificou-se três ações, apontadas como o ato de esquecer os problemas relacionados ao trabalho quando não se encontram na sua atividade laboral, o isolamento diante da situação de sofrimento e o não envolvimento com os idosos para não sofrerem posteriormente com a sua morte (Mariano \& Carreira, 2016).

A comunicação também foi uma estratégia de enfrentamento evidenciada nos estudos. $\mathrm{O}$ trato entre a equipe de enfermagem e com os demais trabalhadores, particularmente com a chefia, mediado pelo diálogo, é uma das peculiaridades da organização de trabalho nas ILPI que fornecem o prazer. O bom relacionamento entre equipe tem resposta positiva na relação do trabalhador de enfermagem e no seu exercício laboral, pois o trabalho em conjunto contribui na superação de situações que originam sofrimento (Mariano \& Baldissera, 2015; Rodrigues et al., 2016).

E por fim, a atividade física segundo o estudo que aborda o perfil dos cuidadores de Itaúna, traz que $76 \%$ dos seus cuidadores realizam atividade física regularmente e que a prática contínua tem repercussão benéfica contra a depressão e ansiedade, alívio de dores, e auxilia na manutenção da saúde mental sendo uma excelente ferramenta contra o estresse (Brasil, 2016).

\section{Considerações Finais}

Conforme o objetivo proposto por esta revisão de escopo, de analisar os fatores que influenciam a qualidade de vida dos trabalhadores de ILPI, este estudo identificou que existem fatores que refletem negativamente e outros positivamente na qualidade de vida de cada trabalhador de ILPI, bem como, algumas estratégias de enfrentamento propostas apresentadas pelos autores para que estes trabalhadores consigam lidar com as situações específicas de sua área.

Torna-se notório o elevado grau de desgaste físico e mental dos trabalhadores que lidam diariamente com o acúmulo de tarefas, escassez de pessoal, situações de sofrimento, morte, idosos com sentimento de abandono por suas famílias, que sobrecarregam a equipe de enfermagem que acompanha por 24 horas estes pacientes.

Com isto, compreende-se que os trabalhadores de ILPI necessitam de atenção, ainda mais quando se refere a sua qualidade de vida. E incentiva-se que mais pesquisas sejam realizadas com esta população a fim de tornar possível o desenvolver de estratégias públicas de saúde como ações e programas que possam ampará-los, estimulando outras áreas como a inovar os meios de superar tais barreiras e valorizar nossos profissionais que se empenham a cada dia por exercitar um trabalho mais justo e humano a cada idoso. 
Desse modo, pesquisas sobre a qualidade de vida relacionada a trabalhadores de ILPIs, tanto da área da saúde ou não, que envolvam uma equipe multidisciplinar, e que incluam por exemplo trabalhadores da higienização e do ramo alimentício são opções interessantes para futuras pesquisas a serem desenvolvidas.

\section{Referências}

Alves, M. B., Menezes, M. R., Felzemburg, R. D. M., Silva, V. A., \& Amaral, J.B. (2017). Instituições de longa permanência para idosos: aspectos físicoestruturais e organizacionais. Escola Anna Nery, 21(4).

Amorim, J. S. C., Mesas, A. E., \& Trelha, C. S. (2018). Fatores associados à ótima capacidade para o trabalho em servidores idosos de uma universidade no Sul do Brasil. Revista brasileira de saúde ocupacional, (43):15.

Andrade, S. M. B., \& Reis, L. A. (2018). Repercussões do toque terapêutico na qualidade de vida de cuidadores formais de idosos. Fisioterapia Brasil, 19(2):196201.

Arksey, H., \& O'malley L. (2005). Scoping studies: Towards a methodological framework. International Journal Of Social Research Methodology: Theory \& Practice, Heslington, (1):19-32.

Barbosa, L. M., Noronha, K., Spyrides, M. H. C., \& Araújo, C. A. D. (2017). Qualidade de vida relacionada à saúde dos cuidadores formais de idosos institucionalizados em Natal, Rio Grande do Norte. Revista brasileira de estudos populacionais, 34(02).

Brasil. (2016). Conselho Nacional do Ministério Público (CNMP). Estômago. In:Conselho Nacional Do Ministério Público (Brasil). Manual de atuação funcional: O Ministério Público na Fiscalização das Instituições de Longa Permanência para Idosos. Conselho Nacional do Ministério Público.

Brasil. (2005). Ministério da Saúde. Agência Nacional de Vigilância Sanitária (ANVISA). Resolução no $283 / 2005$.

Brasil. (2013) Lei no 12.853 de agosto de 2013. Altera a legislação sobre direito autoral. Diário Oficial da República Federativa do Brasil.

Colomé, I. C. S., Marqui, A. B. T., Jahn, A. C., Resta, D. G., Carli, R., Winck, M. T., \& Nora, T. T. D. (2011). Cuidar de idosos institucionalizados: características e dificuldades dos cuidadores. Revista Eletrônica Enfermagem, 13(2):306-12.

Lopes, L. A., Mitre, N. C. D., \& Coelho, M. A. C. M. (2012). Perfil dos cuidadores das instituições de longa permanência para idosos de Itaúna - MG. ConScientiae Saúde, 11(2):):338-344.

Mariano, P. P., Baldissera, V. D. A., Martins, J. T., \& Carreira, L. (2015). Organização do trabalho de enfermagem em instituições de longa permanência para idosos: relação com o prazer e o sofrimento no trabalho. Texto contexto enferm., 24(3):756-65.

Mariano, P. P., \& Carreira, L. (2016). Estratégias defensivas no ambiente laboral da enfermagem nas instituições de longa permanência para idosos. Rev Gaúcha Enferm, 37(3):e58587.

Mariano, P. P., \& Carreira, L. (2016). Prazer e sofrimento no cuidado ao idoso em instituição de longa permanência: percepção dos trabalhadores de enfermagem. Esc. Anna Nery, 20(4):e20160088.

Mendes, E. A., Teixeira, L. R., \& Bonfatti, R. J. (2017). As condições de saúde dos trabalhadores a partir dos exames periódicos de saúde. Saúde Debate, (41):142-154.

Oliveira, P. B., \& Tavares, D. M. S. (2014). Condições de saúde de idosos residentes em Instituição de Longa Permanência segundo necessidades humanas básicas. Revista Brasileira de Enfermagem, (67):241-6.

Organização Mundial da Saúde. (2015). Relatório mundial de envelhecimento e saúde. http://bvsms.saude.gov.br/bvs/publicacoes/envelhecimento_ativo.pdf.

Pimenta, F. A. P., Alves, R. L., Oliveira, F. L. P., Nascimento, N. R. M., Coelho, G. L. L. M., \& Freitas, S. N. (2019). Qualidade de vida e excesso de peso em trabalhadores em turnos alternantes. Revista brasileira de saúde ocupacional, (44):2.

Rodrigues, C. P., Amorim, J. S. C, Cicero, A. C., Alves, L. A., Fernandes, K. B. P., \& Trelha, C.S. (2016). Estresse e qualidade de vida em técnicos e auxiliares de enfermagem em instituições de longa permanência para idosos. O Mundo da Saúde, 40(2):180-188.

Santos, S. M. C., Oliveira, L. S., Santos, M. C. S., Carneiro, S. R., Santos, L. L. S., \& Teodori, R. M. (2016). Avaliação da sobrecarga de trabalho e da qualidade de vida de cuidadores de idosos institucionalizados. Fisioterapia Brasil, 18(4):433-4.

Vasconcelos, R., Marion, S. R., Pereira, M. C. K., Trindade, L., Toledo, S. V., Colomé, L., \& Beck, C. (2017). Saúde do trabalhador de serviços especializados de saúde mental: revisão de literatura. SIEPE. https://periodicos.unipampa.edu.br/index.php/SIEPE/article/view/98797

Veras, R. P., \& Oliveira, M. (2018). Envelhecer no Brasil: a construção de um modelo de cuidado. Ciência \& saúde coletiva, 23(6): $1929-1936$. 